CARTA AL EDITOR

\section{PERCEPCIÓN SOBRE LA ENFERMEDAD DE CHAGAS Y EL RIESGO DE TRANSMISIÓN ORAL EN ANDOAS, LORETO, PERÚ}

\section{PERCEPTION ABOUT CHAGAS DISEASE AND THE RISK OF ORAL TRANSMISSION IN ANDOAS, LORETO, PERU}

\author{
Rufino Cabrera (1) 1,2,a, Yadira Valderrama (1) 1,b, \\ Juan Ramón Meza (ib) \\ 1 Centro Nacional de Epidemiología, Prevención y Control de Enfermedades \\ (CDC), Ministerio de Salud, Lima, Perú. \\ 2 Escuela de Medicina, Facultad de Ciencias de la Salud, Universidad \\ Peruana de Ciencias Aplicadas, Lima, Perú. \\ 3 Red de Salud Datem del Marañón, Dirección Regional de Salud \\ Loreto,Loreto, Perú.

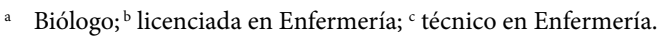

Sr Editor: En Brasil, Venezuela, Colombia, Bolivia ${ }^{(1)}$ y Guayana Francesa ${ }^{(2)}$ se han reportado brotes por transmisión oral de la enfermedad de Chagas asociados epidemiológicamente al consumo de bebidas como el jugo de açaí (fruto de una palmera) y caña de azúcar ${ }^{(1)}$. Entre el 2002 a 2012, se reportaron 959 casos de enfermedad de Chagas aguda (ECA), de ellos, el $66,5 \%$ fueron por transmisión oral, con una tendencia al incremento ${ }^{(1)}$.

En Perú, entre el 2006 a 2010 fueron reportados siete casos de ECA en la cuenca amazónica ${ }^{(3-4)}$, seis de los cuales fueron niños de comunidades nativas de la provincia Datem del Marañon, en el departamento de Loreto. Uno de ellos, fue una niña Candoshi de dos años de la comunidad de Chambira, en el distrito de Andoas ${ }^{(4)}$. En este caso, además de la investigación entomológica, serológica y la entrevista a los padres para establecer si se infectó por vía vectorial, oral o congénita ${ }^{(4)}$, se realizó una encuesta exploratoria de conocimientos, actitudes y prácticas a los jefes de familia de las comunidades de Chambira, San Fernando y Progreso, en mayo de 2010.

Dada la relevancia y la escasa información del riesgo de la transmisión oral, describimos los hallazgos. El objetivo fue

Citar como: Cabrera R, Valderrama Y, Meza JR. Percepción sobre la enfermedad de Chagas y el riesgo de transmisión oral en Andoas, Loreto, Perú. Rev Peru Med Exp Salud Publica. 2019;37(1):174-5. Doi: https://doi.org/10.17843/rpmesp.2020.371.4875

Correspondencia: Rufino Cabrera; Escuela de Medicina, Universidad Peruana de Ciencias Aplicadas. Av. Alameda San Marcos, Cuadra 2, Chorrillos, Lima, Perú; pcmercab@upc.edu.pe

Recibido: 09/10/2019 Aprobado: 30/12/2019 En línea: 23/03/2020 conocer la percepción sobre la enfermedad de Chagas e identificar las bebidas y los jugos que consume la población que son consumidas por la población y que podrían tener potencial riesgo de transmisión oral de Trypanosoma cruzi (T. cruzi).

Las 19 preguntas aplicadas se distribuyeron en datos sociodemográficos (cinco preguntas), conocimientos y hábitos del vector (tres), transmisión (una), participación comunitaria (dos), actitudes (dos), tipo de jugos o bebidas que son consumidos y los recipientes utilizados en su preparación (tres), medidas de prevención (una) y fuentes de información (dos). Para identificar si reconocían los triatominos hematófagos, se les enseñó muestrarios adultos de Panstrongylus geniculatus y de Rhodnius prolixus.

La comunidad de Chambira es eminentemente Candoshi y está ubicada en la ribera del río Huitoyacu, afluente del río Pastaza. En cambio, San Fernando está conformado, principalmente por mestizos y algunos nativos y está ubicado en la unión de los ríos Huitoyacu y Pastaza. Progreso está ubicado entre San Fernando y Chambira.

Fueron entrevistados, previo consentimiento de los cuatro Apus (líderes) de las comunidades, 34 jefes de familia, de los cuales, el 67,6\% (23/34) fueron varones. Una persona de 61 años manifestó haber sido picado, alguna vez, por una "punduna», presentando chagoma y fiebre. Ninguno de los entrevistados conocía el rol de los triatominos en la transmisión de T. cruzi (Tabla 1).

El 52,9\% (18/34) de los entrevistados provenían de San Fernando, 44,1\% (15/34) de Chambira y un jefe de familia (2,9\%) de Progreso. El 94,1\% (32/34) manifestó que aceptaría que le tomen una muestra de sangre para hacerse un examen y descartar alguna infección.

Además, fueron identificados 11 bebidas o jugos ingeridos por la población y que son preparados y conservados en recipientes de boca ancha. Los brotes ocurrirían por el ingreso ocasional de triatominos a las viviendas, atraídas por la luz. El açaí se contaminaría directamente con las heces de los vectores o por triatominos infectados caídos accidentalmente a los recipientes con pulpa de açaí o durante la manipulación o colección del fruto ${ }^{(5)}$.

En un brote por transmisión oral en la Amazonía brasileña, se identificó T. cruzi linaje TcIV, tanto en los pacientes como en el jugo de açaí, confirmándose como agente etiológico de la enfermedad de Chagas ${ }^{(6)}$. Asimismo, el jugo de caña de azúcar, consumido en las comunidades encuestadas, ha sido vinculado, epidemiológicamente, a los brotes de la enfermedad de Chagas ${ }^{(1)}$. En cuanto al riesgo, el «chapo», «mingado de arroz» y la «chicha» son consumidos inmediatamente después de ser hervidos; sin embargo, el resto son bebidas naturales mantenidas en condiciones ambientales; por lo tanto, existe el riesgo de su potencial contaminación si se mantienen en recipientes abiertos. 
Tabla 1. Conocimientos de los jefes de familia sobre triatominos, preparación de jugos o bebidas y medidas de prevención para evitar picadura de insectos, Andoas, Loreto, 2010

\begin{tabular}{|c|c|}
\hline Variables & $\mathrm{n} / \mathrm{N}(\%)$ \\
\hline Edad, mediana (RIC) & $38(20-26)$ \\
\hline \multicolumn{2}{|l|}{ Comunidad nativa } \\
\hline Candoshi & $22 / 34(64,7)$ \\
\hline Cocama-Camilla & $1 / 34(2,9)$ \\
\hline Colono & $9 / 34(26,4)$ \\
\hline \multicolumn{2}{|l|}{ Nivel educativo } \\
\hline Sin educación & $8 / 34(23,5)$ \\
\hline Primaria & $18 / 34(52,9)$ \\
\hline Secundaria & $5 / 34(14,7)$ \\
\hline Superior & $3 / 34(8,8)$ \\
\hline \multicolumn{2}{|l|}{ Nombre del triatomino } \\
\hline Punduna (en Candoshi) & $16 / 34(47,1)$ \\
\hline Chinche & $12 / 34(35,3)$ \\
\hline No sabe & $6 / 34(17,6)$ \\
\hline \multicolumn{2}{|l|}{ Lugar donde vio a los triatominos a } \\
\hline Dormitorio & $11 / 34(32,3)$ \\
\hline Palmeras & $2 / 34(5,8)$ \\
\hline Debajo de troncos o árboles & $2 / 34(5,8)$ \\
\hline Peridomicilio / gallinero & $2 / 34(5,8)$ \\
\hline En áreas de cultivo ${ }^{\mathrm{b}}$ & $11 / 34(32,3)$ \\
\hline \multicolumn{2}{|l|}{ Alimentación de los triatominos ${ }^{a}$} \\
\hline Sangre de perro, gallina, hombre, mono & $3 / 34(8,8)$ \\
\hline No sabe & $25 / 34(73,5)$ \\
\hline \multicolumn{2}{|l|}{ Jugos o bebidas que consumen } \\
\hline Masato (bebida fermentada de yuca) & $34 / 34(100,0)$ \\
\hline Chapo (plátano maduro cocinado) & $20 / 34(58,8)$ \\
\hline Jugo de aguaje (pulpa con agua y azúcar) & $18 / 34(52,9)$ \\
\hline Caña de azúcar (extracto) & $12 / 34(35,3)$ \\
\hline Ungurawi (pulpa del fruto de palmera ungurawi) & $12 / 34(35,3)$ \\
\hline Refresco & $12 / 34(35,3)$ \\
\hline $\begin{array}{l}\text { Mingado de arroz (arroz hervido con agua y } \\
\text { azúcar) }\end{array}$ & $8 / 34(23,5)$ \\
\hline Coco (agua de coco) & $7 / 34(20,5)$ \\
\hline Papaya & $5 / 34(14,7)$ \\
\hline Piña & $3 / 34(8,8)$ \\
\hline Chicha (bebida a base de maíz morado) & $3 / 34(8,8)$ \\
\hline \multicolumn{2}{|l|}{ Recipiente utilizado en la preparación del masato } \\
\hline Olla & $18 / 34(52,9)$ \\
\hline Paila & $9 / 34(26,4)$ \\
\hline Balde & $6 / 34(17,6)$ \\
\hline Jarra & $1 / 34(2,9)$ \\
\hline \multicolumn{2}{|l|}{ Recipiente utilizado para la fermentación del masato } \\
\hline Balde & $20 / 34(58,8)$ \\
\hline Paila & $6 / 34(17,6)$ \\
\hline Olla & $5 / 34(14,7)$ \\
\hline Tacho & $2 / 34(5,8)$ \\
\hline Tina & $1 / 34(2,9)$ \\
\hline \multicolumn{2}{|l|}{ Participaría si se implementa la vigilancia comunal } \\
\hline Sí 1 & $33 / 34(97,1)$ \\
\hline \multicolumn{2}{|l|}{ Lugar para recibir información de salud } \\
\hline Promotor de salud & $20 / 34(58,8)$ \\
\hline A través de la radio comunal & $14 / 34(41,2)$ \\
\hline Profesor & $1 / 34(02,9)$ \\
\hline \multicolumn{2}{|l|}{ Medidas de prevención contra insectos } \\
\hline Uso de mosquiteros & $34 / 34(100)$ \\
\hline Humo de comején & $6 / 34(17,6)$ \\
\hline Uso de insecticida comercial en spray & $5 / 34(14,7)$ \\
\hline Uso de petróleo & $1 / 34(2,9)$ \\
\hline
\end{tabular}

a Se excluyeron a los que no identificaron a los triatominos

${ }^{\mathrm{b}}$ Cultivos de plátano y de camote

RIC: rango intercuartilico
En Perú, la ingesta de aguaje podría ser un vehículo para la transmisión oral de T. cruzi ${ }^{(7)}$. Asimismo, en Datem del Marañón, dos hermanos huambisas con ECA tenían una semana de diferencia en el inicio de sus síntomas, por lo que se habrían infectado por vía oral, al ingerir masato ${ }^{(4)}$. Se requieren estudios de supervivencia o viabilidad de T. cruzi para conocer mejor el riesgo.

El uso de mosquiteros y las alternativas tradicionales podrían ser factores protectores para evitar infectarse con T. cruzi. En una comunidad huambisa, un caso agudo resultó ser el único de su familia que no dormía bajo mosquitero ${ }^{(4)}$.

A pesar de no haberse confirmado brotes por transmisión oral de la enfermedad de Chagas en Perú, en las capacitaciones de los microscopistas en el marco del Plan Malaria Cero, se debería incluir el diagnóstico diferencial de T. cruzi.

Estos resultados preliminares, tiene limitaciones como la antigüedad de los datos y el reducido tamaño muestral, sin embargo, brindamos una primera aproximación al riesgo de transmisión oral de T. cruzi en una comunidad de la Amazonía peruana.

Conflictos de interés: Los autores declaran que no tienen conflicto de interés que reportar.

Contribuciones de los autores: RC y YV participaron en la concepción y diseño del artículo, la recolección de los datos, el análisis e interpretación de datos, la redacción del artículo y la aprobación de la versión final. JRM participó en la recolección de datos, redacción del artículo y aprobación de la versión final.

Financiamiento: Centro Nacional de Epidemiología, Prevención y Control de Enfermedades (CDC) del Ministerio de Salud del Perú.

\section{REFERENCIAS BIBLIOGRÁFICAS}

1. Andrade DV, Gollob KJ, Dutra WO. Acute chagas disease: new global challenges for an old neglected disease. PLoS Negl Trop Dis. 2014;8(7):e3010. doi: 10.1371/journal.pntd.0003010

2. Blanchet $D$, Brenière SF, Schijman AG, Bisio M, Simon S, Véron V, et al. First report of a family outbreak of Chagas disease in French Guiana and posttreatment follow-up. Infect Genet Evol. 2014;28:245-50. doi: 10.1016/j. meegid.2014.10.004

3. Náquira VC, Cabrera R. Breve reseña histórica de la enfermedad de Chagas, a cien años de su descubrimiento y situación actual en el Perú. Rev Med Exp Salud Publica. 2009; 26:496-506.

4. Cabrera R, Vega S, Valderrama Y, Cabanillas CK, Fernández C, Rodríguez $\mathrm{O}$, et al. New focus of active transmission of Chagas disease in indigenous populations in Peruvian Amazon Basin. Rev Soc Bras Med Trop. 2013;46(3):367-72. doi: 10.1590/0037-8682-1195-2013

5. Coura JR, Junqueira AC, Fernandes O, Valente SA, Miles MA. Emerging Chagas disease in Amazonian Brazil. Trends Parasitol. 2002;18(4):171-6.

6. Santana RAG, Guerra MGVB, Sousa DR, Couceiro K, Ortiz JV, Oliveira $\mathrm{M}$, et al. Oral transmission of Trypanosoma cruzi, Brazilian Amazon. Emerg Infect Dis. 2019;25(1):132-35. doi: 10.3201/eid2501.180646

7. Cabrera R. Notas breves sobre Psammolestes tertius, Bergroth, 1911 (Reduviidae: Hemiptera): un triatomino silvestre. An Fac Med. 2006;67(4):345-6. 